\title{
Curcumin ameliorates mitochondrial dysfunction associated with inhibition of gluconeogenesis in free fatty acid-mediated hepatic lipoapoptosis
}

\author{
JONG-JEN KUO ${ }^{1,2}$, HEN-HONG CHANG ${ }^{1-3}$, TUNG-HU TSAI ${ }^{1}$ and TZUNG-YAN LEE ${ }^{3}$ \\ ${ }^{1}$ Institute of Traditional Medicine, National Yang Ming University, Taipei; ${ }^{2}$ Center for Traditional Chinese \\ Medicine, Chang Gung Memorial Hospital, Taoyuan; ${ }^{3}$ Graduate Institute of Traditional \\ Chinese Medicine, Chang Gung University, Taoyuan, Taiwan, R.O.C.
}

Received March 13, 2012; Accepted May 18, 2012

DOI: $10.3892 /$ ijmm.2012.1020

\begin{abstract}
Insulin resistance occurs in almost all patients with non-alcoholic fatty liver disease (NAFLD), and mitochondrial dysfunction likely plays a pivotal role in the progression of fatty liver into non-alcoholic steatohepatitis (NASH). Curcumin is a compound derived from the spice turmeric, a spice that is a potent antioxidant, anti-carcinogenic, and anti-hepatotoxic agent. The aim of this study was to analyze the ability of curcumin to protect against the mitochondrial impairment induced by high free fatty acids (HFFAs) and to determine the underlying mechanism for this cytoprotection. Curcumin treatment inhibited the lipoapoptosis, ROS production and ATP depletion elicited by HFFA in primary hepatocytes. We demonstrate that curcumin effectively suppressed HFFA-induced production of phosphoenol pyruvate carboxykinase (PEPCK) and glucose-6-phosphatase (G6Pase) in hepatocytes. Not only did curcumin treatment increase mitochondrial DNA (mtDNA) copy number in hepatocytes, but it also increased levels of transcriptional factors that regulate mitochondrial biogenesis, including peroxisome proliferator-activated receptor- $\gamma$ coactivator $1 \alpha(\mathrm{PGC} 1 \alpha)$, nuclear respiratory factor 1 (NRF1) and mitochondrial transcription factor A (Tfam). In addition, curcumin contributed to cell survival, as indicated by the restoration of the mitochondrial membrane potential (MMP) and the inhibition of the mitochondrial biogenesis induced by HFFA. Furthermore, this cytoprotection resulted from curcumin-mediated downregulation of the NF- $\mathrm{kB}$ p65 subunit, thereby inhibiting lipoapoptosis. Together, these data suggest that curcumin protects hepatocytes from HFFA-induced lipoapoptosis and mitochondrial dysfunction, which partially occurs through the regulation of mitochondrial biogenesis.
\end{abstract}

Correspondence to: Professor Tzung-Yan Lee, Graduate Institute of Traditional Chinese Medicine, Chang Gung University, 259 Wen-Hwa First Road, Kwei-Shan, Taoyuan 333, Taiwan, R.O.C.

E-mail: joyamen@mail.cgu.edu.tw

Key words: curcumin, mitochondrial dysfunction, lipoapoptosis

\section{Introduction}

Non-alcoholic fatty liver disease (NAFLD) has emerged as a substantial public health concern. This syndrome is characterized by steatosis in hepatocytes and elevated serum levels of free fatty acids (FFAs) (1). Although the mechanisms responsible for fatty liver are not fully elucidated, an increased delivery and transport of FFAs into the liver, and augmented hepatic fatty acid synthesis are likely to play a significant role in the pathogenesis of NAFLD. Liver cell apoptosis is a prominent feature of NAFLD and correlates with disease severity (2). The toxicity of lipids, or lipotoxicity, and specifically, lipid-induced apoptosis, or lipoapoptosis, is a potential mechanism relating apoptosis to NAFLD. Hepatocellular lipid accumulation is thought to simultaneously stimulate mitochondrial fatty acid oxidation and the production of ROS, thereby promoting lipid peroxidation and damage of mitochondrial DNA (mtDNA) (3). Abnormal morphological changes in liver mitochondria have been observed in patients and animal models with non-alcoholic steatohepatitis (NASH) (4). There is also growing evidence that FFA-mediated oxidative stress contributes significantly to mitochondrial dysfunction in the liver. Therefore, designing therapies that prevent mitochondrial dysfunction stands to be one of the most important strategies in for treating of NAFLD and its complications.

Despite the high prevalence of NAFLD and its potential to cause serious liver injury, the current therapies for NAFLD are limited. Hence, developing new therapeutic intervention is a prerequisite in the treatment of NAFLD. Curcumin has recently received attention as a promising dietary supplement for liver protection (5). These recent studies showed that curcumin inhibited HSCs activation, and suppressed hepatic fibrogenesis in vitro and in vivo $(6,7)$. In addition, curcumin eliminated the effects of leptin on the activation of HSCs in vitro by reducing the phosphorylation level of the leptin receptor (Ob-R), leading to the suppression of $\mathrm{Ob}-\mathrm{R}$ gene expression and the interruption of leptin signaling (8). Curcumin also exerts potential anti-inflammatory effects in diverse cell types, including pancreatic cells, chondrocytes, and hepatic cells $(9,10)$. Wang et al (11) have illustrated curcumin's ability to reduce pro-inflammatory cytokines in 3T3-L1 adipocytes 
with FFA-induced insulin resistance. Although these suppressive effects of pro-inflammatory signaling pathways might be involved in the pathogenesis of lipotoxicity, there is no evidence that curcumin is an anti-lipoapoptosis agent against FFA-induced mitochondrial dysfunction in hepatocytes.

The overall objectives of this study were to examine whether curcumin has a protective effect on HFFA-induced lipoapoptosis in hepatocytes and to explore the possibility of a mechanistic link between oxidative stress and mitochondrial dysfunction. We showed that HFFA-induced obvious lipoapoptosis and mitochondrial dysfunction in primary hepatocytes. Following curcumin treatment, mitochondrial morphology and biogenesis, as well as the mtDNA copy number, were altered. Decreased levels of intracellular ROS and attenuated loss of the mitochondrial membrane potential (MMP) may have contributed to the hepatoprotection.

\section{Materials and methods}

Cell culture. Hepatocyte isolation was performed as described previously (12). Hepatocytes were seeded at $5 \times 10^{6} /$ dish in $10-\mathrm{cm}$ culture dishes, and used at 70-80\% confluence. The cells were maintained at $37^{\circ} \mathrm{C}$ with humidified air in a $5 \% \mathrm{CO}_{2} / 95 \%$ air atmosphere. Hepatocytes were incubated with HFFA, which were prepared according to a slightly modified method described by Kohli et al (13). Curcumin (purity $>94 \%$ ) was purchased from Sigma (St. Louis, MO). Confluent cells were incubated in HFFA medium and with or without curcumin with the appropriate experimental conditions for the indicated time points.

Cellular triglyceride assay and Oil-Red $O$ staining. Cellular lipids were extracted according to the method described by Folch et al (14). The intracellular triglyceride content was measured with a diagnostic triglyceride reagent kit according to the manufacturer's instructions (Antrim, UK). After treatment, cells were washed with ice-cold PBS twice and fixed in $4 \%$ paraformaldehyde for $15 \mathrm{~min}$. The cells were rinsed with $50 \%$ isopropanol once and stained with Oil-Red O for $15 \mathrm{~min}$. Finally, the coverslips were washed and analyzed using light microscopy. Hematoxylin was used as a counterstain.

RNA isolation and real-time PCR analyses. Total-RNA was extracted from the hepatocytes using the guanidinium-phenolchloroform method. Total-RNA $(5 \mu \mathrm{g})$ was reverse-transcribed using the RevertAid ${ }^{\mathrm{TM}}$ First Strand cDNA Synthesis kit according to the manufacturer's protocol. The cDNA was amplified using the TaqDNA polymerase kit (Vilnius, Lithuania). RT-PCR products were separated by electrophoresis on a $3 \%$ agarose gel and were quantified by the ImageQuant 5.2 software (Healthcare Bio-Sciences, Philadelphia, PA). Real-time PCR was performed with a LightCycler 1.5 apparatus (Roche Diagnostics, Mannheim, Germany) using the LightCycler FastStart DNA MasterPLUS SYBR-Green I kit according to the manufacturer's protocol. Mitochondrial DNA copy number was determined by real-time PCR as previously described (15).

Western blotting. Cell lysates were separated by SDS-PAGE and transferred onto a PVDF membrane. The membrane was blocked with blocking buffer containing $5 \%$ non-fat milk, $50 \mathrm{mM}$ Tris (pH 7.6), $150 \mathrm{mM} \mathrm{NaCl}$ and $0.1 \%$ Tween-20
(TBS-T) for $1 \mathrm{~h}$ at room temperature. The membrane was then incubated with various primary antibodies for $8 \mathrm{~h}$ at $4^{\circ} \mathrm{C}$ and subsequently with HRP-linked secondary antibodies for $1 \mathrm{~h}$ at room temperature. The signals were detected using an ECL kit and were quantified by the ImageQuant 5.2 software.

Mitochondria mass and DNA assay and mitochondrial respiratory complexes and ATP measurement. Mitochondria mass were detected according to the method of Tedesco et al (16). Hepatocytes seeded onto glass coverslips were incubated with 100 nM MitoTracker Red 580 (Invitrogen, Carlsbad, CA). After incubation for $30 \mathrm{~min}$ at $37^{\circ} \mathrm{C}$, coverslips were rinsed and washed. DAPI was used for nuclear staining. Finally, the cells were fixed in $4 \%$ paraformaldehyde for $15 \mathrm{~min}$ and visualized using a microscope. The activity of the mitochondrial respiratory complex was analyzed as described (17). The ATP content of hepatocytes was measured with the ATP bioluminescence assay kit HS II according to the manufacturer's protocol (Mannheim, Germany). The luminescence value was normalized by protein concentration and the luminescence ratio was compared with the HFFA-free group.

Flow cytometry for ROS and mitochondrial membrane potential assay. Hepatocytes were incubated with $10 \mathrm{M}$ DCFH-DA (Molecular Probes, Inc., Eugene, OR) at $37^{\circ} \mathrm{C}$ for $40 \mathrm{~min}$ before the termination of treatment. Cells were washed and scraped gently with ice-cold PBS. DCF fluorescence was then detected according to the manufacturer's instructions. The mean fluorescence intensity of JC-1 (BD ${ }^{\mathrm{TM}}$ MitoScreen kit) was measured to determine the MMP. Treated cells were collected and resuspended at a concentration of $1 \times 10^{5} / \mathrm{ml}$ in PBS containing $1 \mathrm{~g} / \mathrm{ml} \mathrm{JC}-1$, and were incubated at $37^{\circ} \mathrm{C}$ for $30 \mathrm{~min}$. Samples were analyzed by flow cytometry using a FACSCalibur (BD Biosciences). The data are presented in terms of relative fluorescence percentage.

Immunofluoresence staining. Hepatocytes $\left(1 \times 10^{3}\right.$ cells $)$ were cultured on glass coverslips. After incubation with HFFA and with or without curcumin, the cells were fixed in $4 \%$ paraformaldehyde for $15 \mathrm{~min}$, washed with ice-cold PBS and blocked with $7.5 \%$ normal goat serum for $30 \mathrm{~min}$ at room temperature. After washing with ice-cold PBS twice, the cells were incubated with anti-PEPCK, anti-glucose-6-phosphatase (G6Pase), anti-NF- $\kappa B$ antibodies for $1 \mathrm{~h}$ at room temperature. After washes with ice-cold PBS twice, the cells were incubated with diluted FITC-conjugated secondary antibody for $1 \mathrm{~h}$ at room temperature. In addition, DAPI was used for nuclear staining. The slides were mounted in mounting medium and visualized using a microscope (Olympus, Japan).

Statistical analysis. Data are presented as the mean \pm SEM. The statistical analyses were performed using a one-way analysis of variance followed by the Student Newman-Keuls multiplerange test. $\mathrm{P}<0.05$ denote statically significant differences.

\section{Results}

The results from the Oil-Red O staining and from the intracellular triglyceride assay indicate that fat accumulation was reduced by curcumin compared with $1 \mathrm{mM}$ HFFA alone (Fig. 1A and B). 
A

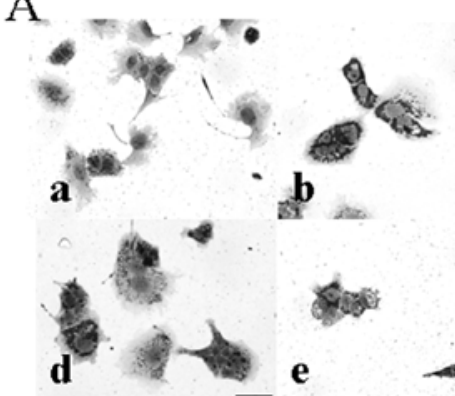

C
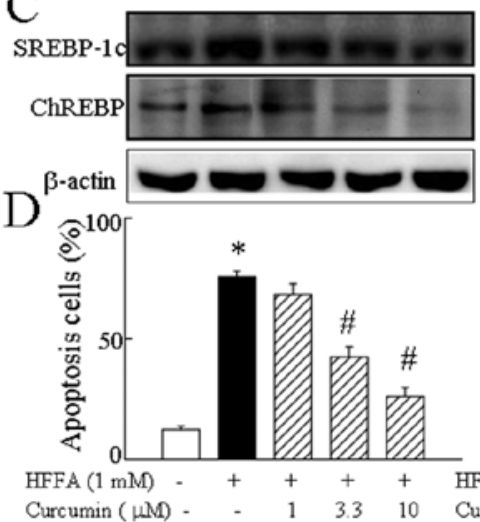

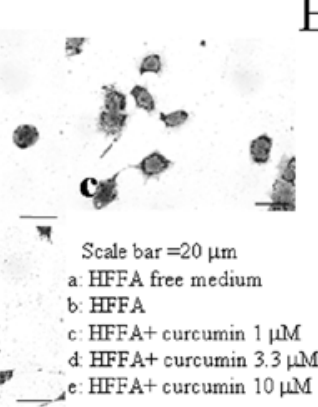

$\mathrm{E}$

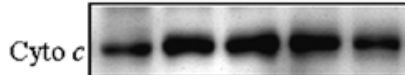

B

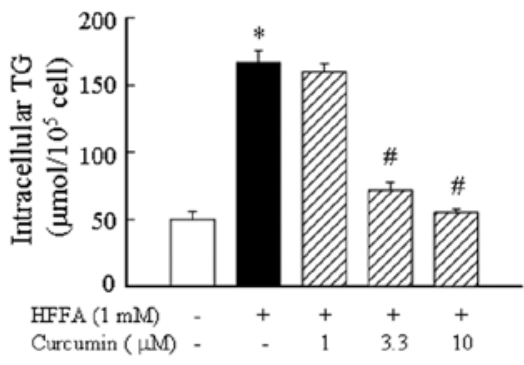

$\mathrm{F}$

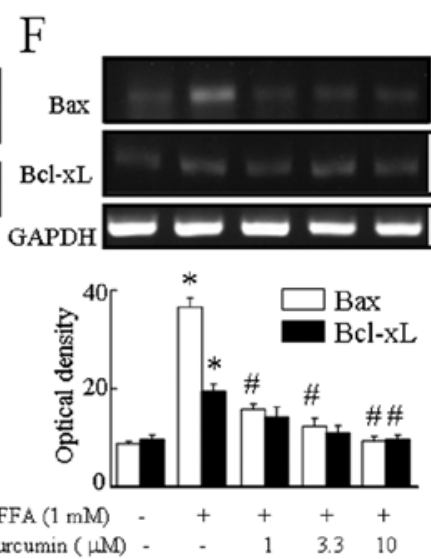

Figure 1. Effects of curcumin on HFFA-mediated lipid accumulation and lipoapoptosis in hepatocytes. (A) Hepatocytes were incubated in curcumin and $1 \mathrm{mM}$ HFFA for $12 \mathrm{~h}$. Intracellular lipid inclusion was examined by Oil-Red O staining. (B) Hepatocytes were co-incubated with $1 \mathrm{mM}$ HFFA and curcumin for $12 \mathrm{~h}$. The lipid accumulation was assessed by a commercial triglyceride reagent kit. (C) The protein levels of SREBP-1 and ChREBP were analyzed using western blot analysis. (D) DAPI-stained apoptotic nuclei were quantitated using fluorescence microscopy. Two hundred random cells were counted for each condition. Cells were stimulated with HFFA in the absence (-) or presence (+) of curcumin at various concentrations for $12 \mathrm{~h}$. Curcumin causes an inhibition of (E) cytochrome $\mathrm{c}$ and $(\mathrm{F})$ Bax and Bcl-xL expression by HFFA. The data are expressed as the mean \pm SEM representative of three independent experiments. ${ }^{*} \mathrm{P}<0.05$ vs. HFFA-free medium; ${ }^{*} \mathrm{P}<0.05$ vs. 1 mM HFFA treatment.

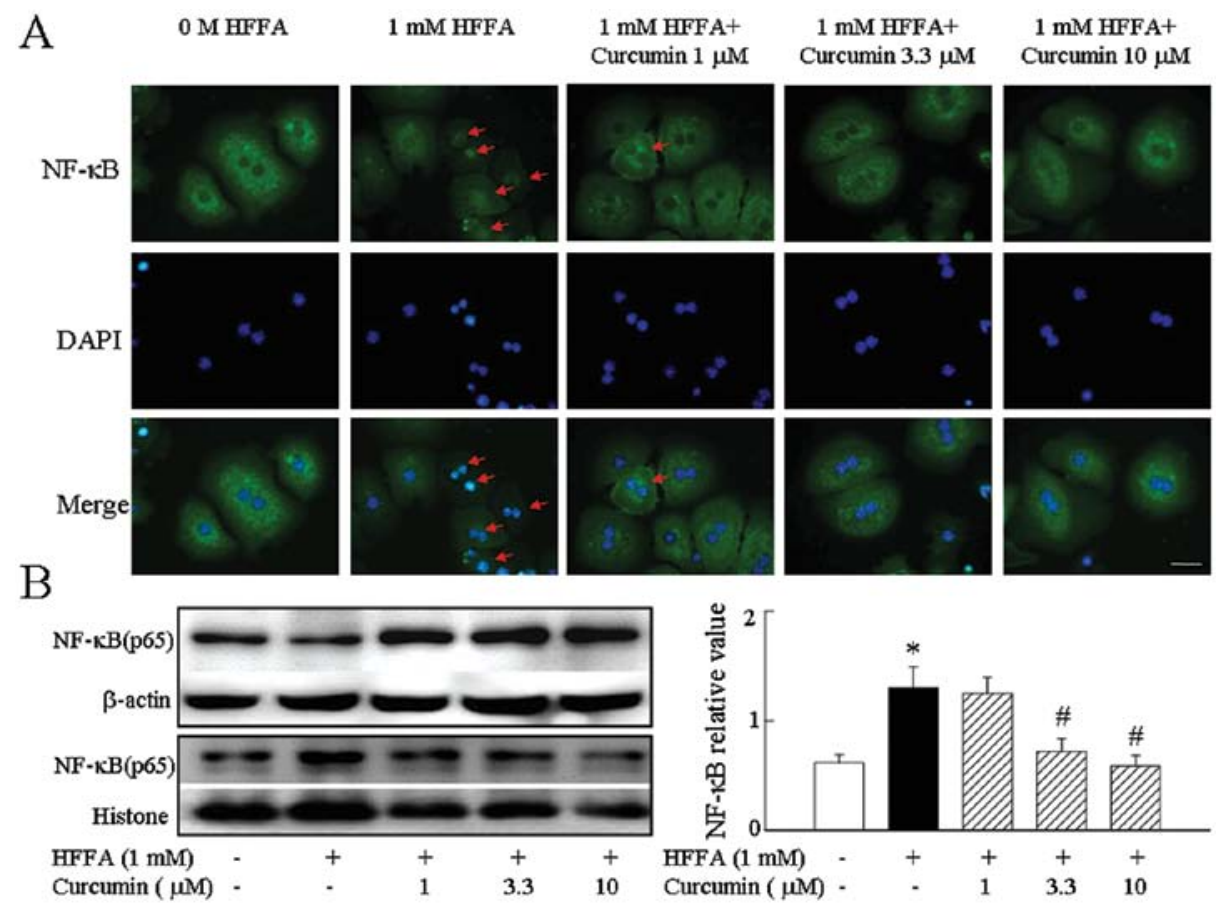

Figure 2. Curcumin reduces NF- $\mathrm{B}$ nuclear translocation in HFFA-stimulated hepatocytes. (A) Curcumin inhibits HFFA-induced nuclear translocation of NF- $\mathrm{KB}$ in hepatocytes, as detected using immunofluorescence. They were then visualized by Alexa Fluor ${ }^{\circledR} 488$ nuclear staining (green) and anti-p65 immunofluorescence to monitor nuclear translocation of $\mathrm{p} 65$. The nuclei of the corresponding cells were visualized by DAPI staining. Staining was analyzed by confocal laser scanning microscopy. Nuclear localization of p65 is visible as yellow staining generated where the color images merge. Total magnification for the images was $\mathrm{x} 40$. (B) Hepatocytes were stimulated with $1 \mathrm{mM}$ HFFA with (+) or without (-) curcumin for $2 \mathrm{~h}$. The amounts of cytosolic NF- $\mathrm{kB}(\mathrm{p} 65)$ and nuclear NF- $\mathrm{kB}$ (p65) were determined by western blot analysis. The relative value of NF-kB (p65) protein is expressed as the band intensity normalized to the intensity of $\beta$-actin or histone protein, respectively. All analyses are representative of three independent experiments. ("P $<0.05$ vs. control; ${ }^{\#} \mathrm{P}<0.05$ vs. HFFA alone). 

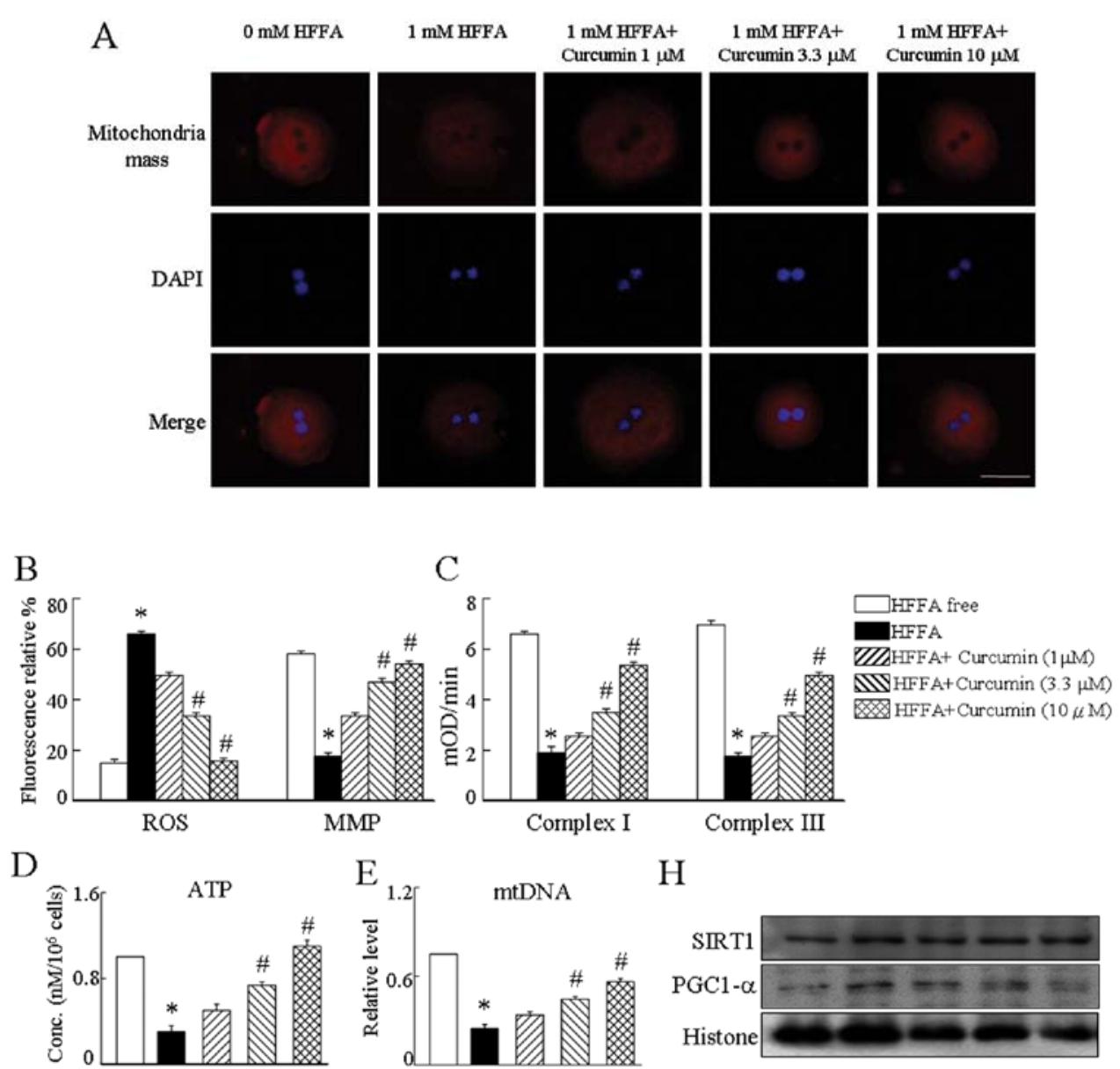

$\mathrm{H}$

$\mathrm{F}$

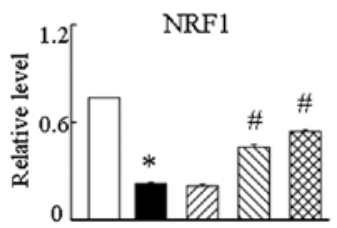

G

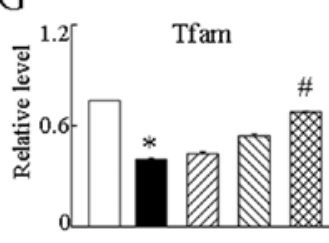

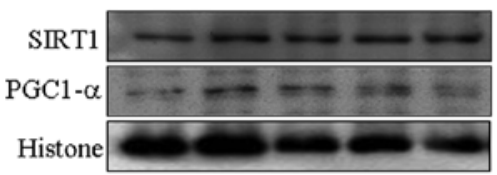

$4[\quad \square$ SIRT1
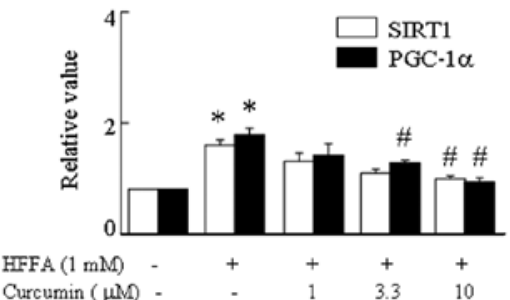

Figure 3. Effects of curcumin on abnormal mitochondrial biogenesis and ROS content in HFFA-stimulated hepatocytes. (A) Mitochondrial mass was visualized as a Mitotracker Red signal by fluorescence microscopy. (B) Cellular ROS production and mitochondrial membrane potential were detected by flow cytometry against DCFH-DA and JC-1 fluorescence, as described in the Materials and methods. (C) The activity of mitochondrial complexes I and III were determined by a spectrophotometrical activity assay. (D) Relative cellular ATP level was analyzed by the ATP bioluminescence assay kit and was normalized to treatment with HFFA-free medium. The mRNA level of (E) mtDNA, (F) NRF1 and (G) Tfam were analyzed by real-time PCR. The mtDNA amount was expressed as mtDNA copy number per nuclear DNA copy number. (H) The nuclear amounts of SIRT1 and PGC-1 $\alpha$ were determined by western blot analysis. The histone was detected as internal control. The data are shown as mean \pm SEM representative of three independent experiments $\left({ }^{*} \mathrm{P}<0.05\right.$ vs. HFFA-free group; " $\mathrm{P}<0.05$ vs. HFFA alone).

This effect was especially visible at $10 \mu \mathrm{M}$ curcumin. SREBP-1c and ChREBP protein levels were clearly suppressed by treatment with $10 \mu \mathrm{M}$ curcumin (Fig. 1C). The results indicated that suppression of the HFFA-mediated increase of cellular triglycerides by curcumin may be associated with downregulation of the expression of lipogenic factors. HFFA treatment significantly increased the percentage of positively stained (i.e. apoptotic) cells to $80 \%$ in cultured hepatocytes, whereas curcumin co-treatment reduced the proportion of apoptotic cells to $30 \%$ in hepatocytes (Fig. 1D). Release of cytochrome c (Fig. 1E) and Bax (Fig. 1F) into the cytosol with HFFA was confirmed by immunoblot or RT-PCR analysis of cytosolic fraction. Curcumin significantly counteracted the HFFA-induced upregulation of apoptosisinducing factors.
We found that the p65 subunit of $\mathrm{NF}-\kappa \mathrm{B}$ was distributed in the cytoplasm in all cells before HFFA stimulation. Treatment with HFFA resulted in a marked accumulation of p65 in nuclei after $2 \mathrm{~h}$. HFFA activated NF- $\kappa \mathrm{B}$ in hepatocytes and curcumin was a potent inhibitor of $\mathrm{NF}-\kappa \mathrm{B}$ activity (Fig. 2A). We further confirmed these results by western blot analysis to probe nuclear and cytoplasmic extracts using an antibody specific for the p65 subunit of NF- $\kappa$ B. Treatment of hepatocytes with curcumin followed by exposure to HFFA significantly inhibited $\mathrm{NF}-\kappa \mathrm{B}$ translocation to the nucleus and returned the p65 subunit of NF- $\kappa \mathrm{B}$ into the cytoplasm (Fig. 2B).

Hepatocytes treated with HFFA showed a significant decrease of the MitoTracker Red signal, indicating the decrease of mitochondrial function compared to HFFA-free cells 
A
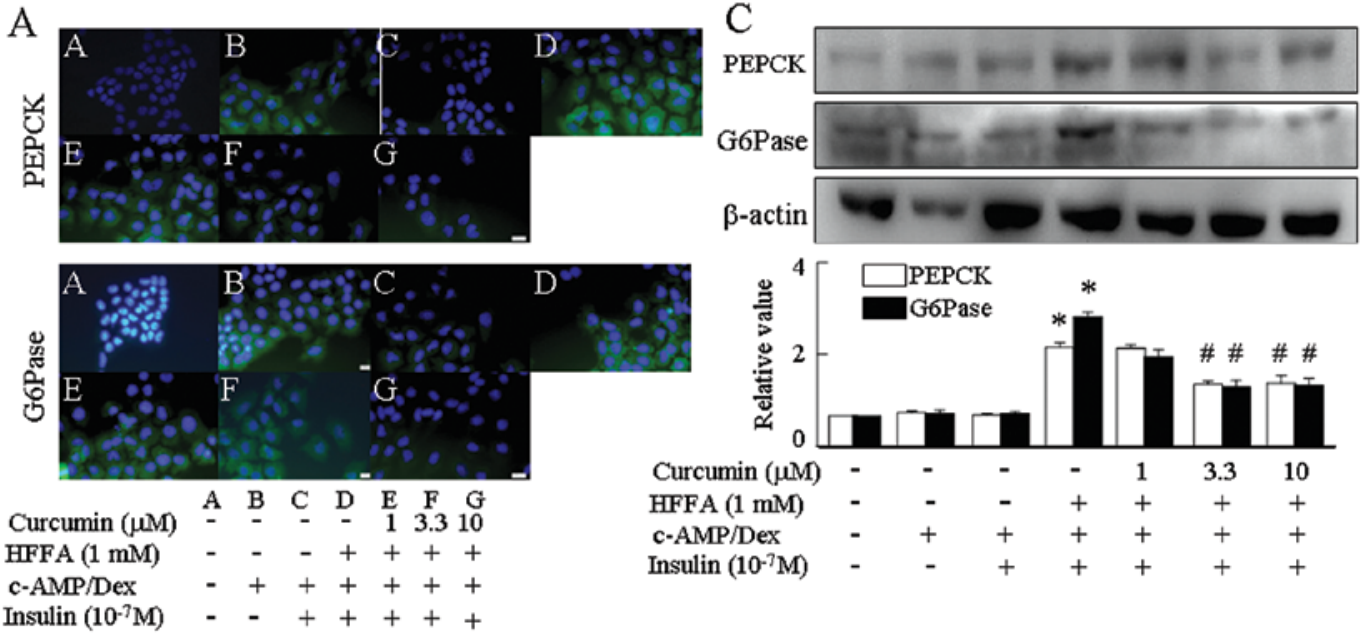

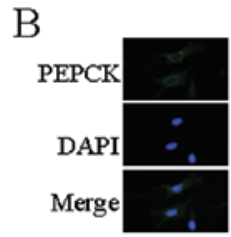

HFFA (1 mM) Curcumin $(\mu \mathrm{M})$

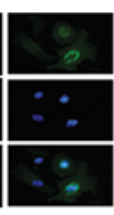

$+$

$-$

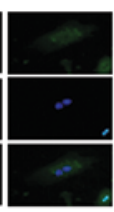

$+$

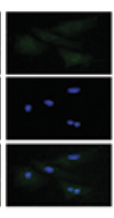

$+$

3.3

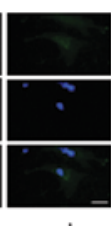

$+$

10

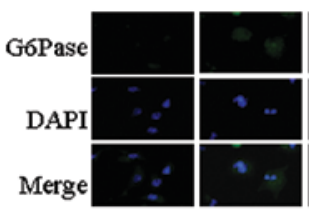

$+$

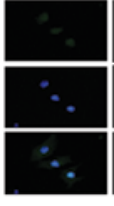

$+$

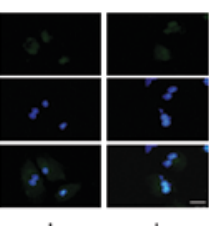

$+$

10

Figure 4. Curcumin ameliorated HFFA-abrogated insulin suppression of PEPCK and G6Pase expression. The protein levels of PEPCK and G6Pase were measured after incubation with curcumin and $1 \mathrm{mM} \mathrm{HFFA}$ for $10 \mathrm{~h}$. Cells were then treated with $10-7 \mathrm{M}$ insulin for 30 min and cAMP (100 $\mu \mathrm{M}$ )/dexamethasone $(500 \mathrm{nM})(\mathrm{c}-\mathrm{AMP} / \mathrm{Dex})$ for $2 \mathrm{~h}$. The protein levels were detected by (A and B) immunofluorescent staining and (C) western blotting. The data were shown as the mean \pm SEM representative of three independent experiments. ( $\mathrm{P}<0.05$ vs. HFFA-free group; ${ }^{*} \mathrm{P}<0.05$ vs. HFFA alone). The immunofluorescent images were shown at $\mathrm{x} 40$ magnification. DAPI was used for nuclear staining.

(Fig. 3A). To verify that the inhibition of mitochondria mass signal by HFFA is a relevant molecular mechanism by which mitochondrial biogenesis is affected, hepatocytes were treated with $1 \mathrm{mM}$ HFFA in the presence of curcumin corcumin prevented the effect of HFFA on mitochondrial biogenesis with a full restoration of ATP levels (Fig. 3D), NRF1, Tfam mRNA expression (Fig. 3F and G), PGC-1 $\alpha$ and SIRT1 protein levels (Fig. 3H), and the mtDNA amount in hepatocytes (Fig. 3E). As mitochondrial biogenesis was affected by treatment with curcumin, we investigated whether these treatments affected mitochondrial respiratory complexes (MRC). We found that complex I and III activities were altered after incubation with curcumin prevented the reduction of MRC in HFFA-treated cells (Fig. 3C). We next sought to determine whether HFFA affects ROS content and MMP. ROS levels in hepatocytes treated with HFFA were much higher than that of control cells. Conversely, HFFA led to a large reduction in MMP. As expected, the results demonstrated that the presence of curcumin suppressed the ROS generation and upregulation of MMP expression in HFFA-treated hepatocytes. These observations suggest that curcumin can attenuate HFFA-induced aspects of mitochondrial dysfunction, including depression of the MMP and production of ROS (Fig. 3B).

The expression of two key hepatic gluconeogenic genes, PEPCK and G6Pase, increased in response to cAMP/dexamethasone (Dex) and was reduced in response to insulin. By immunofluorescence, treatment with $1 \mathrm{mM}$ HFFA abrogated the insulin suppression of cAMP/Dex-mediated PEPCK and G6Pase levels when compared with HFFA-free medium (Fig. 4A and B). Curcumin repressed the expression of PEPCK and G6Pase in a dose-dependent manner in HFFA-induced aspects of gluconeogenesis (Fig. 4C).

\section{Discussion}

NAFLD is characterized by an elevated serum concentration of FFAs, liver steatosis and hepatocyte apoptosis $(1,2)$. Excess free fatty acids may impair normal cell signaling, causing cellular dysfunction or induced lipoapoptosis (18). Others have suggested that mitochondrial dysfunction plays a central role in FFA-induced apoptosis (19). In this study, we demonstrated that curcumin could protect hepatocytes from FFA-induced oxidative damage and could reverse FFA-stimulated lipoapoptosis. The mechanism of action may be related to improvements in mitochondrial function and biogenesis. Our results suggest that curcumin or a similar compound could be used to treat NAFLD or NASH.

Previous study has shown that apoptotic cells undergo mitochondrial perturbations including loss of the MMP and generation of ROS (20). In addition, the redox-sensitive nuclear transcriptional factor, $\mathrm{NF}-\kappa \mathrm{B}$, acts as a proapoptotic factor in the pathogenesis of NAFLD and NF- $\kappa$ B activation plays a critical role in the pathophysiology of cytokine-mediated hypoglycaemia $(21,22)$. It is therefore reasonable to suppose that the interactions between genes of apoptosis and gluconeogenesis are regulated by $\mathrm{NF}-\kappa \mathrm{B}$. In this study, curcumin has been shown to have profound effects on mitochondrial function. Curcumin effectively prevented the loss of MMP in hepatocytes in response to HFFA challenge, suggesting that curcumin either increases the expression of a protein that acts 
to decrease the activity of a caspase that lies upstream of the mitochondria in the apoptotic cascade or decreases activation of the NF- $\kappa \mathrm{B}$ cascade (either directly or indirectly). In fact, curcumin has been shown to act directly on some NF- $\kappa \mathrm{B}$ dependent genes (23). We demonstrate that suppression of $N F-\kappa B$ enhances the ability of curcumin to rescue hepatocytes from HFFA-induced lipoapoptosis, strongly suggesting that a decrease in $N F-\kappa B$ activity by curcumin is at least partially responsible for this rescue of mitochondrial function. Another attractive idea is that curcumin might inhibit the decline in ATP production by affecting energy metabolism in the mitochondria. In the current study, we observed that curcumin inhibited triglyceride accumulation in HFFA-treated hepatocytes. The expression of lipogenic transcription factors (SREBP-1 and ChREBP) was also reduced. Given that ATP is required for the expression and function of the lipogenic factors, restoring the ATP levels provides a potential mechanism to counteract the inhibition of lipogenesis by curcumin.

Abnormalities in lipid metabolism within hepatocytes are still poorly understood. Accumulating evidence indicates that mitochondrial dysfunction plays a central role in the pathogenesis of NAFLD and that NAFLD is a mitochondrial disease (24). A number of mechanisms may explain the mitochondrial dysfunction observed in NAFLD patients. One of these mechanisms is oxidative stress. ROS-induced depletion of mtDNA can affect mitochondrial function and induces hepatic steatosis (25). The HFFA-induced decrease in the mitochondrial respiratory chain (MRC) activity in hepatocytes is partly due to oxidative stress, as treating cells with a potent antioxidant, curcumin, improved the activity of complexes I and III of the MRC. Previous studies showed that the activity of MRC complexes is decreased in liver tissue from patients with NASH (26). In the current study, we confirm that the activity of MRC complexes I and III are reduced by 30 or $70 \%$, respectively. These results were similar to those found in NASH patients and indicate that this in vitro model can be used to investigate the mechanisms of mitochondrial dysfunction.

Insulin resistance and oxidative stress both contribute to the pathogenesis of NAFLD and the progression from steatosis to steatohepatitis (27). High levels of FFA in the plasma increase hepatic FFA uptake, whereas high insulin levels may increase FFA synthesis concomitant with hepatic insulin resistance in some obese individuals (28). In our current results, the promotion of PEPCK and G6Pase expression is associated with increased expression of SREBP-1 and ChREBP in HFFA-treated hepatocytes. These processes demonstrate the close interrelation between gluconeogenesis and hepatic lipid metabolism and indicate that NAFLD involves dysregulation in lipogenesis and gluconeogenesis-mediated hepatic insulin signaling. It is known that PGC-1 $\alpha$ is a coactivator with pleiotropic functions such as controlling mitochondrial biogenesis and function; both functions are vital links in the regulatory network for metabolic homeostasis (29). In our study, curcumin upregulated mitochondrial biogenesis through reversal of HFFA-induced upregulation of PGC-1 $\alpha$ levels, thereby supporting the potential utility of curcumin to improve the metabolic status in patients with fatty liver. For the first time, this study demonstrated a curcumin-mediated increase in the expression of genes regulating hepatic mitochondrial biogenesis, including, PGC-1 $\alpha$, NRF-1 and Tfam, and an increase in the expression of genes that commonly regulate mtDNA content. Additionally, curcumin may be involved directly in the modulation of gluconeogenesis. This observation is supported by previous studies showing that curcumin suppressed gluconeogenesis and glycemic control $(30,31)$. Thus, it is reasonable to hypothesize that a curcumin-induced blockade of lipotoxicity may directly prevent mitochondrial dysfunction and gluconeogenesis. Taken together, our data suggest that curcumin may serve as a potential therapeutic approach to ameliorate the cytotoxicity of HFFA by acting through multiple pathways involved in mitochondrial function.

\section{Acknowledgements}

This study was supported by grant no. CCMP100-RD-016 from the Committee on Chinese Medicine and Pharmacy, Department of Health; grant no. NSC98-2320-B-182-021-MY3 from the National Science Council, Taiwan; and grant no. CMRPD180242 from the Chang Gung Memorial Hospital, Linkuo, Taiwan.

\section{References}

1. Feldstein AE, Canbay A, Guicciardi ME, Higuchi H, Bronk SF and Gores GJ: Diet associated hepatic steatosis sensitizes to Fas mediated liver injury in mice. J Hepatol 39: 978-983, 2003.

2. Feldstein AE, Canbay A, Angulo P, Taniai M, Burgart LJ, Lindor KD and Gores GJ: Hepatocyte apoptosis and fas expression are prominent features of human nonalcoholic steatohepatitis. Gastroenterology 125: 437-443, 2003.

3. Mantena SK, Vaughn DP, Andringa KK, et al: High fat diet induces dysregulation of hepatic oxygen gradients and mitochondrial function in vivo. Biochem J 417: 183-193, 2009.

4. Rao MS and Reddy JK: PPARalpha in the pathogenesis of fatty liver disease. Hepatology 40: 783-786, 2004.

5. O'Connell MA and Rushworth SA: Curcumin: potential for hepatic fibrosis therapy. Br J Pharmacol 153: 403-405, 2008.

6. Fu Y, Zheng S, Lin J, Ryerse J and Chen A: Curcumin protects the rat liver from $\mathrm{CCl}_{4}$-caused injury and fibrogenesis by attenuating oxidative stress and suppressing inflammation. Mol Pharmacol 73: 399-409, 2008.

7. Zheng $\mathrm{S}$ and Chen A: Curcumin suppresses the expression of extracellular matrix genes in activated hepatic stellate cells by inhibiting gene expression of connective tissue growth factor. Am J Physiol Gastrointest Liver Physiol 290: G883-G893, 2006.

8. Tang Y, Zheng S and Chen A: Curcumin eliminates leptin's effects on hepatic stellate cell activation via interrupting leptin signaling. Endocrinology 150: 3011-3020, 2009.

9. Bharti AC, Takada Y and Aggarwal BB: Curcumin (diferuloylmethane) inhibits receptor activator of NF-kappa B ligand-induced NF-kappa B activation in osteoclast precursors and suppresses osteoclastogenesis. J Immunol 172: 5940-5947, 2004.

10. Bachmeier BE, Mohrenz IV, Mirisola V, et al: Curcumin downregulates the inflammatory cytokines CXCL1 and -2 in breast cancer cells via NFkappaB. Carcinogenesis 29: 779-789, 2008.

11. Wang SL, Li Y, Wen Y, Chen YF, LX Na, ST Li and Sun CH: Curcumin, a potential inhibitor of up-regulation of TNF-alpha and IL-6 induced by palmitate in 3T3-L1 adipocytes through NF-kappaB and JNK pathway. Biomed Environ Sci 22: 32-39, 2009.

12. Lee TY, Chen FY, Chang HH and Lin HC: The effect of capillarisin on glycochenodeoxycholic acid-induced apoptosis and heme oxygenase- 1 in rat primary hepatocytes. Mol Cell Biochem 325: 53-59, 2009.

13. Kohli R, Pan X, Malladi P, Wainwright MS and Whitington PF: Mitochondrial reactive oxygen species signal hepatocyte steatosis by regulating the phosphatidylinositol 3-kinase cell survival pathway. J Biol Chem 282: 21327-21336, 2007.

14. Folch J, Lees M and Sloane Stanley GH: A simple method for the isolation and purification of total lipides from animal tissues. $\mathrm{J}$ Biol Chem 226: 497-509, 1957. 
15. Tedesco L, Valerio A, Cervino C, et al: Cannabinoid type 1 receptor blockade promotes mitochondrial biogenesis through endothelial nitric oxide synthase expression in white adipocytes. Diabetes 57: 2028-2036, 2008.

16. Tedesco L, Valerio A, Dossena M, et al: Cannabinoid receptor stimulation impairs mitochondrial biogenesis in mouse white adipose tissue, muscle, and liver: the role of eNOS, p38 MAPK, and AMPK pathways. Diabetes 59: 2826-2836, 2010.

17. González R, Ferrín G, Hidalgo AB, et al: N-acetylcysteine, coenzyme Q10 and superoxide dismutase mimetic prevent mitochondrial cell dysfunction and cell death induced by d-galactosamine in primary culture of human hepatocytes. Chem Biol Interact 181: 95-106, 2009.

18. Schaffer JE: Lipotoxicity: when tissues overeat. Curr Opin Lipidol 14: 281-287, 2003.

19. Wei Y, Rector RS, Thyfault JP and Ibdah JA: Nonalcoholic fatty liver disease and mitochondrial dysfunction. World $\mathrm{J}$ Gastroenterol 14: 193-199, 2008.

20. Hirsch T, Marzo I and Kroemer G: Role of the mitochondrial permeability transition pore in apoptosis. Biosci Rep 17: 67-76, 1997.

21. Li L, Chen L, Hu L, et al: Nuclear factor high-mobility group box 1 mediating the activation of Toll-like receptor 4 signaling in hepatocytes in the early stage of nonalcoholic fatty liver disease in mice. Hepatology 54: 1620-1630, 2011.

22. Liu SF and Malik AB: NF-kappa B activation as a pathological mechanism of septic shock and inflammation. Am J Physiol Lung Cell Mol Physiol 290: L622-L645, 2006.

23. Rafiee P, Binion DG, Wellner M, et al: Modulatory effect of curcumin on survival of irradiated human intestinal microvascular endothelial cells: role of Akt/mTOR and NF-kappaB. Am J Physiol Gastrointest Liver Physiol 298: G865-G877, 2010.
24. Pessayre D and Fromenty B: NASH: a mitochondrial disease. J Hepatol 42: 928-940, 2005.

25. Demeilliers C, Maisonneuve C, Grodet A, et al: Impaired adaptive resynthesis and prolonged depletion of hepatic mitochondrial DNA after repeated alcohol binges in mice. Gastroenterology 123: 1278-1290, 2002

26. Pérez-Carreras M, Del Hoyo P, Martín MA, et al: Defective hepatic mitochondrial respiratory chain in patients with nonalcoholic steatohepatitis. Hepatology 38: 999-1007, 2003.

27. Videla LA, Rodrigo R, Araya J and Poniachik J: Insulin resistance and oxidative stress interdependency in non-alcoholic fatty liver disease. Trends Mol Med 12: 555-558, 2006.

28. Gallagher EJ, Leroith D and Karnieli E: Insulin resistance in obesity as the underlying cause for the metabolic syndrome. Mt Sinai J Med 77: 511-523, 2010.

29. Lin J, Wu H, Tarr PT, et al: Transcriptional co-activator PGC-1 alpha drives the formation of slow-twitch muscle fibres. Nature 15: 797-801, 2002.

30. Weisberg SP, Leibel R and Tortoriello DV: Dietary curcumin significantly improves obesity-associated inflammation and diabetes in mouse models of diabesity. Endocrinology 149: 3549-3558, 2008.

31. Kim T, Davis J, Zhang AJ, He X and Mathews ST: Curcumin activates AMPK and suppresses gluconeogenic gene expression in hepatoma cells. Biochem Biophys Res Commun 388: 377-382, 2009. 